of total $(n=3,941)$ and site-specific fractures (arm, $n=566$; wrist, $n=889$; hip, $n=945$; leg, $n=366$; ankle, $n=520$; other main sites i.e. clavicle, rib and vertebra, $n=467$ ) by diet group over a mean of 17.6 years of follow-up.

Results Compared with regular meat-eaters, vegetarians had marginally higher risks of total fractures (hazard ratios 1.10; $95 \%$ confidence interval 1.00-1.20) and arm fractures (1.28; 1.01-1.63), while vegans had higher risks of total fractures (1.44; 1.21-1.72), arm fractures $(1.60,1.01-2.54)$ and leg fractures $(2.06 ; 1.22-3.47)$. For hip fractures, the risks were significantly higher in fish-eaters $(1.28 ; 1.03-1.59)$, vegetarians $(1.27 ; 1.05-1.55)$ and vegans $(2.35 ; 1.67-3.30)$ compared with regular meat-eaters. There were no significant differences in risks of wrist, ankle or other main site fractures by diet group. Overall, the significant associations appeared to be stronger before adjustment for BMI (e.g. 1.52; 1.27-1.81 in vegans for total fractures), and were slightly attenuated but remained significant with additional adjustment for dietary calcium and total protein.

Conclusion Overall, non-meat eaters, especially vegans, had higher risks of either total or some site-specific fractures, particularly hip fractures, than regular meat eaters. These differences may be partially related to lower BMI or lower dietary intakes of calcium and protein in the non-meat eaters. Further studies of non-European and contemporary populations are needed to determine the generality of these findings.

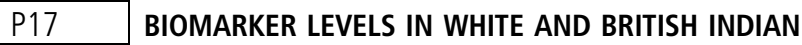 VEGETARIANS AND NON-VEGETARIANS IN THE UK BIOBANK}

${ }^{1}$ TYN Tong*, ${ }^{1}$ A Perez-Cornago, ${ }^{2}$ KE Bradbury, ${ }^{1}$ TJ Key. ${ }^{1}$ Cancer Epidemiology Unit, Nuffield Department of Population Health, University of Oxford, Oxford, UK; ${ }^{2}$ National Institute for Health Innovation, School of Population Health, Faculty of Medical and Health Sciences, University of Auckland, Auckland, New Zealand

\subsection{6/jech-2020-SSMabstracts.113}

Background A comprehensive description of disease biomarker levels in people of different habitual diet groups is lacking. We conducted cross-sectional analyses of mean biomarker concentrations by diet group in a large cohort.

Methods The UK Biobank recruited around 500,000 middle aged participants throughout the United Kingdom in 20062010. Blood and urine samples were collected from the majority of participants, and assayed for a range of serum and urinary biomarkers related to disease status of six outcomes (cardiovascular diseases, bone and joint health, cancer, diabetes, renal disease, and liver). Using multivariable linear regression adjusted for age, sex, fasting status, body mass index and lifestyle confounders, we estimated geometric mean biomarkers concentrations by six diet groups (221,295 regular meat-eaters, 222,038 low meat-eaters, 5,053 poultry eaters, 10,470 fish eaters, 6,804 vegetarians, 416 vegans) in white British participants, and two diet groups in British Indian participants (4091 meat eaters, 1444 vegetarians).

Results We observed differences in the concentrations of many biomarkers by diet group. The biomarkers with the largest percentage difference by extreme diet groups (i.e. vegans versus regular meat-eaters) within each disease outcome group in the white British population are reported below. Compared with white British regular meat-eaters, white British vegans had lower C-reactive protein $(1.10,1.00-1.21$ versus 1.43 , $1.43-1.44 \mathrm{mg} / \mathrm{L})$ and low density lipoprotein cholesterol (3.12, 3.06-3.19 versus $3.65,3.65-3.65 \mathrm{mmol} / \mathrm{L})$; lower vita$\min \mathrm{D}(34.3,33.0-35.8$ versus $44.5,44.4-44.5 \mathrm{nmol} / \mathrm{L})$; higher sex hormone-binding globulin $(51.1,48.9-53.3$ versus 45.0, 44.9-45.0 nmol/L); lower haemoglobin A1C (HbA1C, $33.8,33.4-34.2$ versus $35.2,35.2-35.2 \mathrm{mmol} / \mathrm{mol}$ ); lower urinary creatinine $(5389,5076-5723$ versus 7289, 7269-7308 $\mu \mathrm{mol} / \mathrm{L})$; and lower gamma glutamyltransferase (23.4, 22.124.7 versus 29.7, 29.6-29.8 U/L). Patterns were similar in British Indians, with the exception of HbA1C which was not significantly different between meat-eaters and vegetarians. In both ethnicities, the differences in biomarker levels by diet group were consistent between men and women.

Conclusion In this large population cohort, participants of different diet groups exhibited differences in many biomarkers. These biomarkers are associated with disease risk, and therefore the observed differences may be suggestive of differences in future disease risks by diet group, which should be further investigated.

\section{P18 STRUCTURAL ASPECTS OF SOCIAL CONNECTEDNESS AND ADHERENCE TO A HEART-HEALTHY DIET IN 60-64 YEAR OLDS IN THE NATIONAL SURVEY OF HEALTH AND DEVELOPMENT (NSHD)}

C Constable Fernandez*, J Maddock, P Patalay. MRC Lifelong Health and Ageing, University College London, London, UK

\subsection{6/jech-2020-SSMabstracts. 114}

Background Social connectedness has been shown to influence mortality to the same extent as other well-established risk factors such as smoking. Structural aspects of social connectedness include martial, cohabitation and employment status which are the focus of the present study. Dietary behaviour is a possible pathway through which social relationships exert their influence on health outcomes. Among those above 65 years, research has indicated that living alone and being socially isolated is a risk factor for poor diet variety. The association between retirement and diet quality is not well established, and at 60-64 years, the present study group represent an interesting time of transition in the life course. Moreover, evidence suggests social influences on dietary behaviour are sex-specific. This study aims to examine the association of structural aspects of social networks and diet quality in the 60-64 years age group.

Methods Participants came from the Medical Research Council National Survey of Health and Development (NSHD). The cohort has been followed up 24 times from birth, including a follow-up in 2006-10 at ages 60-64 years when 1,869 participants completed 5 -day prospective estimated diet diaries. Diet quality was assessed according to adherence of a Dietary Approaches to Stop Hypertension (DASH)-type diet score ranging from 0-40. Participants were asked, via questionnaire, to report their current marital status, the number of people living in their household and their current employment status.

Linear regression models were used to determine if marital status, the number of people living in the participant's household and employment status were associated with adherence to a DASH-type diet. Models were also stratified by sex. 